\title{
Independent prognostic role of human papillomavirus genotype in cervical cancer

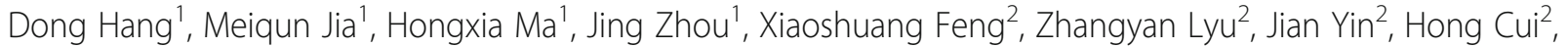
Yin Yin ${ }^{1}$, Guangfu Jin ${ }^{1}$, Zhibin Hu ${ }^{1,3}$, Hongbing Shen ${ }^{1,3}$, Kai Zhang $^{4^{*}}, \mathrm{Ni} \mathrm{Li}^{2^{*}}$ (D) and Min Dai ${ }^{2^{*}}$

\begin{abstract}
Background: Although the correlation of HPV genotype with cervical precursor lesions and invasive cancer has been confirmed, the role of HPV genotype in cervical cancer prognosis is less conclusive. This study aims to systematically investigate the independent prognostic role of HPV genotype in cervical cancer.

Methods: A total of 306 eligible patients provided cervical cell specimens for HPV genotyping before therapy and had a median follow-up time of 54 months after diagnosis. Survival times were measured from the date of diagnosis to the date of cervical cancer-related death (overall survival, OS) and from the date of diagnosis to the date of recurrence or metastasis (disease free survival, DFS). Log-rank tests and Cox proportional hazard models were performed to evaluate the association between HPV genotype and survival times.

Results: A total of 12 types of high-risk HPV were detected and the leading ten types belong to two species: alpha-9 and alpha-7. HPV16 and 18 were the two most common types, with the prevalence of $60.8 \%$ and $8.8 \%$, respectively. In the univariate analysis, HPV16-positive cases were associated with better OS $(P=0.037)$ and HPV16related species alpha-9 predicted better OS and DFS (both $P<0.01$ ). After adjusting for age, FIGO stage, and therapy, HPV16 showed a hazard ratio (HR) of $0.36(95 \% \mathrm{Cl}: 0.18,0.74 ; P=0.005)$ for OS, and alpha-9 resulted in a HR of 0.17 (95\% Cl: $0.08,0.37 ; P<0.001$ ) for OS and 0.32 (95\% Cl: $0.17,0.59 ; P<0.001$ ) for DFS.
\end{abstract}

Conclusions: HPV genotype poses differential prognoses for cervical cancer patients. The presence of HPV16 and its related species alpha-9 indicates an improved survival.

Keywords: Cervical cancer, Prognosis, Human papillomavirus, Genotype

\section{Background}

Cervical cancer is the fourth most common malignancy in females worldwide, with an estimated 527,600 new cases and 265,700 deaths per year [1]. The etiological relationship has been well established between human papillomavirus (HPV) and cervical cancer. Recently, more than $170 \mathrm{HPV}$ genotypes have been identified and classified according to their L1 open reading frame [2]. When HPVs have 60-70\% genomic nucleotide similarity, they are clustered into the same species. Two HPV

\footnotetext{
* Correspondence:

kai87968@sina.com; nli@cicams.ac.cn; daimin2002@hotmail.com

${ }^{4}$ Department of Cancer Prevention, National Cancer Centre/Cancer Hospital, Chinese Academy of Medical Sciences and Peking Union Medical College, Beijing 100021, China

2Program Office for Cancer Screening in Urban China, National Cancer Centre/Cancer Hospital, Chinese Academy of Medical Sciences and Peking Union Medical College, Beijing 100021, China

Full list of author information is available at the end of the article
}

species, alpha-7 (HPV18, 39, 45, 59, 68, and 70) and alpha-9 (HPV16, 31, 33, 35, 52, 58, and 67), are responsible for over $80 \%$ of all cervical cancer cases [3].

Although there has been much evidence on the role of HPV genotype in cervical precursor lesions and invasive cancer, it remains unclear whether they affect prognosis of cervical cancer. Furthermore, existing results on the relationship of HPV genotype with survival are heterogeneous. For example, early evidence showed that HPV16 positivity predicted poor prognosis and was associated with histological features of prognostic significance such as squamous cell carcinomas, pelvic node metastases, and lymphatic space invasion [4]. But some studies reported that HPV18 positivity, rather than HPV16, is a poor prognostic factor $[5,6]$. Histologic type of adenocarcinomas, pelvic lymph node metastasis, and deeper stromal invasion was more common in HPV18-caused 
cervical cancer [6]. In addition, HPV31-related and HPV58-related types were found to be associated with better survival outcome $[7,8]$. However, no prognostic value of HPV type was reported by the other studies $[9,10]$. The inconsistency may be attributed to the significant differences in sample size, length of follow-up, assay methods, and adjustment for known prognostic factors.

To better understand the role of HPV genotype in prognosis of patients with cervical cancer, we assessed the association of HPV genotype with overall survival (OS, the time between the date of diagnosis and the date of cervical cancer-related death) and disease free survival (DFS, the time between the date of diagnosis and the date of recurrence, distant metastasis, or the last followup) among 306 cases of cervical cancer from China.

\section{Methods}

\section{Patients}

Cervical cancer patients were consecutively recruited from Cancer Hospital, Chinese Academy of Medical Sciences from 2010 to 2012. We included patients who had a first diagnosis of histologically confirmed invasive cervical cancer, and the sampling of cervical exfoliated cells for HPV genotyping were taken by a gynecologist before therapy. Patients were excluded for the following criteria: a history of hysterectomy or conization, recurrent cervical cancer, other preexisting malignancies, and those with less than two months of survival after completing therapy. Patient's age, FIGO stage, tumor histology, and pathologic variables were retrieved from medical records. This study was approved by the ethics committees of National Cancer Centre/Cancer Hospital, Chinese Academy of Medical Sciences, and all patients provided informed written consent before study enrollment.

\section{HPV genotyping}

Genomic DNA was extracted from cervical cell specimens manually by using QIAamp DNA Mini Kit, according to the manufacture's protocol (Qiagen, Valencia, CA, USA). The quality of extracted DNA was assessed by PCR with a set of primers for the housekeeping gene $\beta$-actin (forward primer, 5'-GAAATCGTGCGTGACATTAA-3'; reverse primer 5' -AAGGAAGGCTGGAAGA.

GTG-3'). All $\beta$-actin positive specimens were tested for HPV DNA by following the manufacturer's protocol of a HPV GenoArray Test Kit (HybriBio, Beijing, China), which is a Chinese FDA-approved assay for HPV genotyping. A total of $21 \mathrm{HPV}$ types could be detected simultaneously, including 13 high-risk (HR) types (HPV16, 18, 31, 33, 35, 39, 45, 51, 52, 56, 58, 59, and 68), two intermediate-risk types (HPV53 and 66), and six low-risk HPV types (HPV6, 11, 42, 43, 44, and 81).

\section{Treatment and follow up}

Treatment information was retrieved from medical records and was summarized and grouped as follows: surgery alone (radical hysterectomy and pelvic lymphadenectomy); surgery plus adjunctive chemotherapy (CT), radiotherapy (RT) or chemoradiotherapy (CRT); concurrent chemoradiotherapy (CCRT); CT or RT only. Each patient was followed up every 3 months in the first year and every 6 months in the next years by personal or family contacts, until June 2016. Hospital medical records were obtained in order to confirm the reported events. Only validated events were included in analysis. Overall survival (OS) was defined as the time between the date of diagnosis and the date of cervical cancer-related death or the last follow-up. Disease-free survival (DFS) was measured from the date of diagnosis to the date of recurrence, distant metastasis, or the last follow-up.

\section{Statistical analysis}

The data were analyzed by using the Stata version 11.0 (Stata Corporation, Texas, USA). To assess the potential of HPV type as a prognostic biomarker for cervical cancer patients with no matter single or multiple infections, all cases were included for HPV16 and 18 survival analysis. Multiple infections with only alpha-9 types were included for alpha-9 survival analysis. Survival curves were generated using the Kaplan-Meier method, and comparisons were performed using the log-rank test. Multivariate analyses of the factors associated with OS and DFS were done using Cox proportional hazard regression model. In the stratified analysis, the chisquare test-based Q-statistic was applied to test the heterogeneity between subgroups defined by age, FIGO stage, and treatment. All $P$ values presented were twosided and were assumed significant as $P<0.05$.

\section{Results}

\section{Patient characteristics}

The demographic and clinical characteristics of cervical cancer patients are summarized in Table 1. This study included 306 women with a median age of 48 years (range: 26-71 years). The most common histological type was squamous cell carcinoma (96.7\%) and the others were adenocarcinoma (AC) and adeno-squamous carcinoma (ASC). Most of patients were diagnosed with FIGO stage I-II (81.0\%). Seventy-eight patients $(25.5 \%)$ received surgery, and $21(6.9 \%)$ surgery plus CT/RT/ CRT, 160 (52.3\%) CCRT, 47 (15.4\%) CT or RT only.

\section{HPV genotypes}

A total of 12 types of high-risk HPV were detected in this study (Table 2). The top three types were HPV16 (60.8\%), $18(8.8 \%)$, and $52(5.9 \%)$. In the leading ten types, five (HPV16, 52, 33, 31, 58) were alpha-9 and the 
Table 1 Characteristics of cervical cancer patients

\begin{tabular}{|c|c|c|}
\hline \multirow[t]{2}{*}{ Characteristics } & \multicolumn{2}{|c|}{ Patients $(N=306)$} \\
\hline & No. & $\%$ \\
\hline \multicolumn{3}{|l|}{ Age, years } \\
\hline Median & 48 & \\
\hline Range & $26-71$ & \\
\hline$<40$ & 45 & 14.71 \\
\hline $40-50$ & 133 & 43.46 \\
\hline$>50$ & 128 & 41.83 \\
\hline \multicolumn{3}{|l|}{ Histologic type ${ }^{a}$} \\
\hline SCC & 296 & 96.73 \\
\hline AC/ASC & 10 & 3.26 \\
\hline \multicolumn{3}{|l|}{ FIGO stage } \\
\hline । & 105 & 34.31 \\
\hline$\|$ & 143 & 46.73 \\
\hline III & 52 & 16.99 \\
\hline IV & 6 & 1.96 \\
\hline \multicolumn{3}{|l|}{ Differentiation } \\
\hline Poor & 87 & 28.43 \\
\hline Moderate & 126 & 41.18 \\
\hline Well & 11 & 3.59 \\
\hline Unclassified & 82 & 26.80 \\
\hline \multicolumn{3}{|l|}{ Treatment $^{b}$} \\
\hline Surgery & 78 & 25.49 \\
\hline Surgery plus CT/RT/CRT & 21 & 6.86 \\
\hline CCRT & 160 & 52.29 \\
\hline $\mathrm{CT}$ or RT only & 47 & 15.36 \\
\hline
\end{tabular}

aSCC squamous cell carcinoma, $A C$ adenocarcinoma, ASC adeno-squamous carcinoma

${ }^{\mathrm{b}} C T$ chemotherapy, $R T$ radiotherapy, $C R T$ chemoradiotherapy,

$C C R T$ concurrent chemoradiotherapy

others (HPV18, 39, 59, 68, 45) were alpha-7. Of 306 patients, 268 (87.6\%) harbored single-type and 38 (12.4\%) contained multiple-type infections. As shown in Additional file 1: Table S1, HPV16 (81.6\%, 31/38) and 52 $(42.1 \%, 16 / 38)$ were the predominant types that made up the multiple infections.

\section{Survival analysis}

The mean number of follow-up was 5 for each patient and the median time for these follow ups was 54 (range, $3-75)$ months. A total of 58 patients (19.0\%) had experienced treatment failure, including 27 recurrences and 38 distant metastases (7 patients had both). In addition, 34 deaths $(11.1 \%)$ were attributed to cervical cancer. The 5year OS rate for the entire cohort was $87.1 \%$ (95\% CI: $82.1-90.8 \%$ ), and the corresponding DFS rate was $78.3 \%$ (95\% CI: 72.5-83.1\%).

In univariate analysis (Table 3), FIGO stage IV was significantly associated with poorer OS $(P<0.001)$ and
DFS $(P<0.001)$, while primary surgical treatment was associated with a better OS $(P=0.004)$ and DFS $(P=0.019)$. Of note, patients infected with HPV16 had a better OS than those with any other types $(P=0.037)$ (Fig. 1a). HPV16-related species alpha-9 also posed a better OS $(P<0.001)$ and DFS $(P=0.005)$, compared to alpha-7 (Fig. 1b and c). No significant association with prognosis was found for HR-HPV multiple infections, HPV18 and the other types.

In multivariate models with adjustment for patients' age, FIGO stage, and treatment, HPV16 was independently associated with better OS $(\mathrm{HR}=0.36$, 95\% CI: $0.18,0.74 ; P=0.005$ ) (Table 3 ). Infection with the five alpha-9 types was independently associated with better OS $(\mathrm{HR}=0.17,95 \%$ CI: $0.08-0.37 ; P<0.001)$ and DFS $(\mathrm{HR}=0.32,95 \% \mathrm{CI}: 0.17-0.59 ; P<0.001)$. Further analysis suggested that HPV52/33/31/58 group had more favorable OS $(\mathrm{HR}=0.12,95 \% \mathrm{CI}: 0.02-0.57$; $P=0.008)$ and DFS (HR $=0.21,95 \% \mathrm{CI}: 0.06,0.77$; $P=0.018$ ) (Additional file 1: Table S2). Given the limitation of statistical power, we did not find meaningful results when analyzing these four types individually.

To better understand the effect of HPV genotype on cervical cancer survival, stratified analyses based on age, FIGO stage, and treatment were performed. Although the protective effects of HPV16 and alpha-9 were more evident among those with FIGO stage III/IV and those receiving primary $\mathrm{RT}$ and/or $\mathrm{CT}$, no significant difference was detected between subgroups (homogeneity test $P>0.05$ for all) (Additional file 1: Table S3).

\section{Discussion}

Despite recent progress in multimodal treatments, the clinical outcome of cervical cancer remains unfavorable. TNM or FIGO classification based on cervical pathology has insufficient predictive ability, because significant differences in survival are often observed for the same stage. Thus, it is highly necessary to explore additional biomarkers for the identification of a more effective therapeutic strategy against cervical cancer. In this study, we investigated prognostic value of HPV genotype for patients with cervical cancer. A total of $12 \mathrm{HR}$ types were identified and HPV16 positivity was independently associated with lower risk of cervical cancer death than the group of the other $11 \mathrm{HR}$ types. In addition, alpha-9 species including five $\operatorname{HR}$ types $(16,52,33,31$, and 58) was a predictor of better survival compared with alpha-7 species group including the other five HR types $(18,39$, 59,68 , and 45).

Substantial differences in risk for high-grade cervical intraepithelial neoplasia (CIN) and cervical cancer have been revealed between HR HPV types, in which HPV16 and HPV18 confer the highest risk $[11,12]$ However, the relationship between HPV genotype and cervical cancer 
Table 2 Distribution of HR-HPV types in cervical cancer patients

\begin{tabular}{|c|c|c|c|c|c|}
\hline HR-HPV type ${ }^{a}$ & & Species & $\begin{array}{l}\mathrm{SCC}^{\mathrm{b}} \\
(N=296)\end{array}$ & $\begin{array}{l}{\mathrm{AC} / \mathrm{ASC}^{\mathrm{C}}}(\mathrm{N}=10)\end{array}$ & Total (\%) \\
\hline \multirow[t]{13}{*}{ Single infection } & & & & & $268(87.58)$ \\
\hline & 16 & alpha-9 & 184 & 2 & $186(60.78)$ \\
\hline & 18 & alpha-7 & 24 & 3 & $27(8.82)$ \\
\hline & 52 & alpha-9 & 17 & 1 & $18(5.88)$ \\
\hline & 39 & alpha-7 & 8 & 0 & $8(2.61)$ \\
\hline & 33 & alpha-9 & 6 & 0 & $6(1.96)$ \\
\hline & 31 & alpha-9 & 5 & 0 & $5(1.63)$ \\
\hline & 58 & alpha-9 & 4 & 1 & $5(1.63)$ \\
\hline & 59 & alpha-7 & 4 & 0 & $4(1.31)$ \\
\hline & 68 & alpha-7 & 4 & 0 & $4(1.31)$ \\
\hline & 45 & alpha-7 & 3 & 0 & $3(0.98)$ \\
\hline & 51 & alpha-5 & 1 & 0 & $1(0.33)$ \\
\hline & 56 & alpha-10 & 1 & 0 & $1(0.33)$ \\
\hline Multiple infection & & & 35 & 3 & $38(12.42)$ \\
\hline Alpha-7 only & & & 43 & 3 & 46 (15.03) \\
\hline Alpha-9 only & & & 237 & 4 & 241 (78.76) \\
\hline
\end{tabular}

${ }^{a} H R-H P V$ high risk human papillomavirus

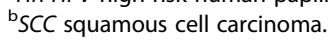

${ }^{\mathrm{c}} A C$ adenocarcinoma, $A S C$ adeno-squamous carcinoma

prognosis has been controversial. Plich et al. identified HPV16 infection as a poor prognostic factor in 204 patients treated by primary radical hysterectomy and pelvic lymphadenectomy [4]. Conversely, another study showed that HPV16 positivity was significantly associated with improved prognosis in the whole series of cervical $\mathrm{AC} /$ ASC and also in subgroup receiving primary RT/CCRT [13]. In our study, the results supported the hypothesis that HPV16 has a favorable impact on the prognosis of cervical cancer. Further, we demonstrated that HPV16related alpha-9 species significantly lowers the risk of cervical cancer-related death and recurrence/metastasis than the alpha-7 species, which was consistent with a previous study in patients undergoing primary radiotherapy [14]. Moreover, although several studies found that HPV18 positivity was associated with poorer prognosis of patients receiving primary surgery $[5,15,16]$, other studies $[17,18]$ and ours failed to support the relationship. Given the much lower prevalence of HPV18 in cervical cancer than HPV16, independent studies with large sample size are needed to assess the impact of HPV18 on patients' prognosis. In addition, because the HPV genotyping kit used in this study does not cover HPV67 and 70, which are high-risk types for cervical cancer, the impact of HPV67 and 70 on prognosis remains to be determined.

The underlying mechanisms that result in the tumors caused by HPV16 and the alpha- 9 species being less aggressive are still undetermined. Interestingly, HPV status has been recognized as a strong and independent factor for favorable survival of patients with oropharyngeal cancer (OPC) $[19,20]$. According to a systematic review, HPV prevalence was $35.6 \%$ (95\% CI: $32.6-38.7 \%$ ) in OPC specimens, and HPV16 accounted for a larger majority of HPV-positive OPC (86.7\%; 95\% CI, 82.6-90.1\%) [21]. A better response to chemotherapy and radiation was observed for HPV-positive OPC [22-24]. In a worldwide survey of HPV genotype in cervix cancer, $61 \%$ of tumors were positive for HPV16 and 83\% were positive for the alpha- 9 species [3], similar to the data in our study. In vitro studies have revealed significant differences in biological behaviors between HPV types. For example, HPV16 is associated with a higher level of tumor apoptosis than HPV18, affording one possible explanation for more radiosensitive cervical cancer with HPV16 [25]. In addition, HR-HPV E6 proteins could interact with cellular PDZ domain-containing proteins to promote cell immortalization, invasion, and epithelial-tomesenchymal transition (EMT) characteristics [26, 27]. There are significant differences in the interactions of HPV16 and HPV18 E6 with the PDZ domain-containing proteins, because a critical difference exists in the amino acid residue at the PDZ-binding motifs of the two E6 proteins [28]. This difference exists not only between HPV16 and HPV18, but also between the alpha-9 and alpha-7 species. Whether the variation in the PDZ domainbinding capacities determines the observed differential therapeutic response is worth additional exploration. 


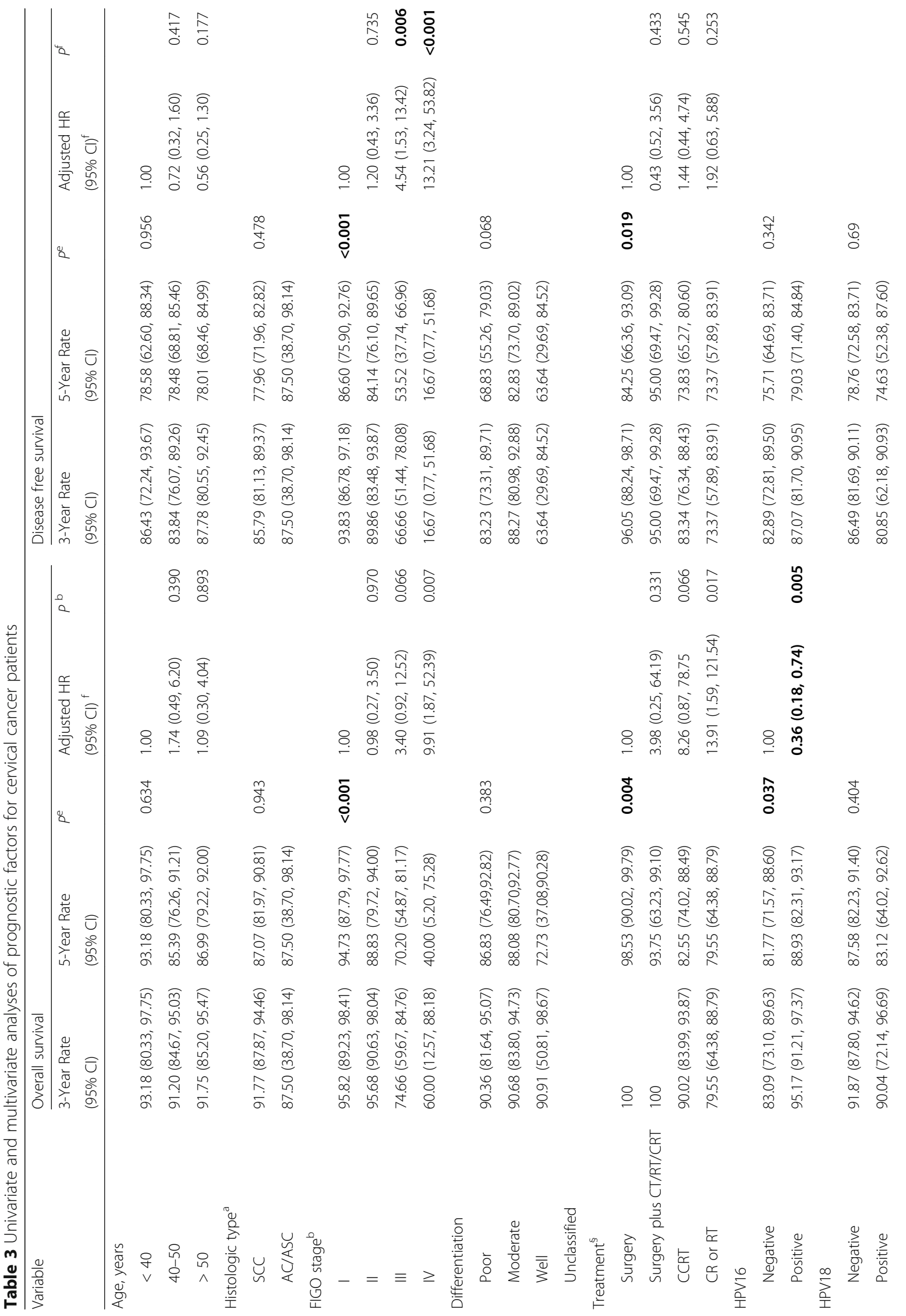




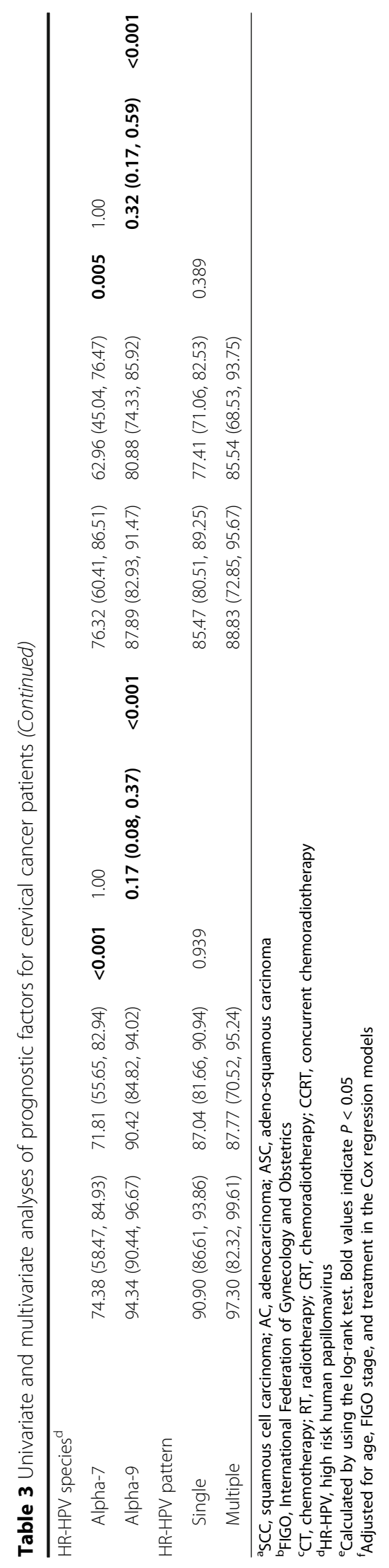



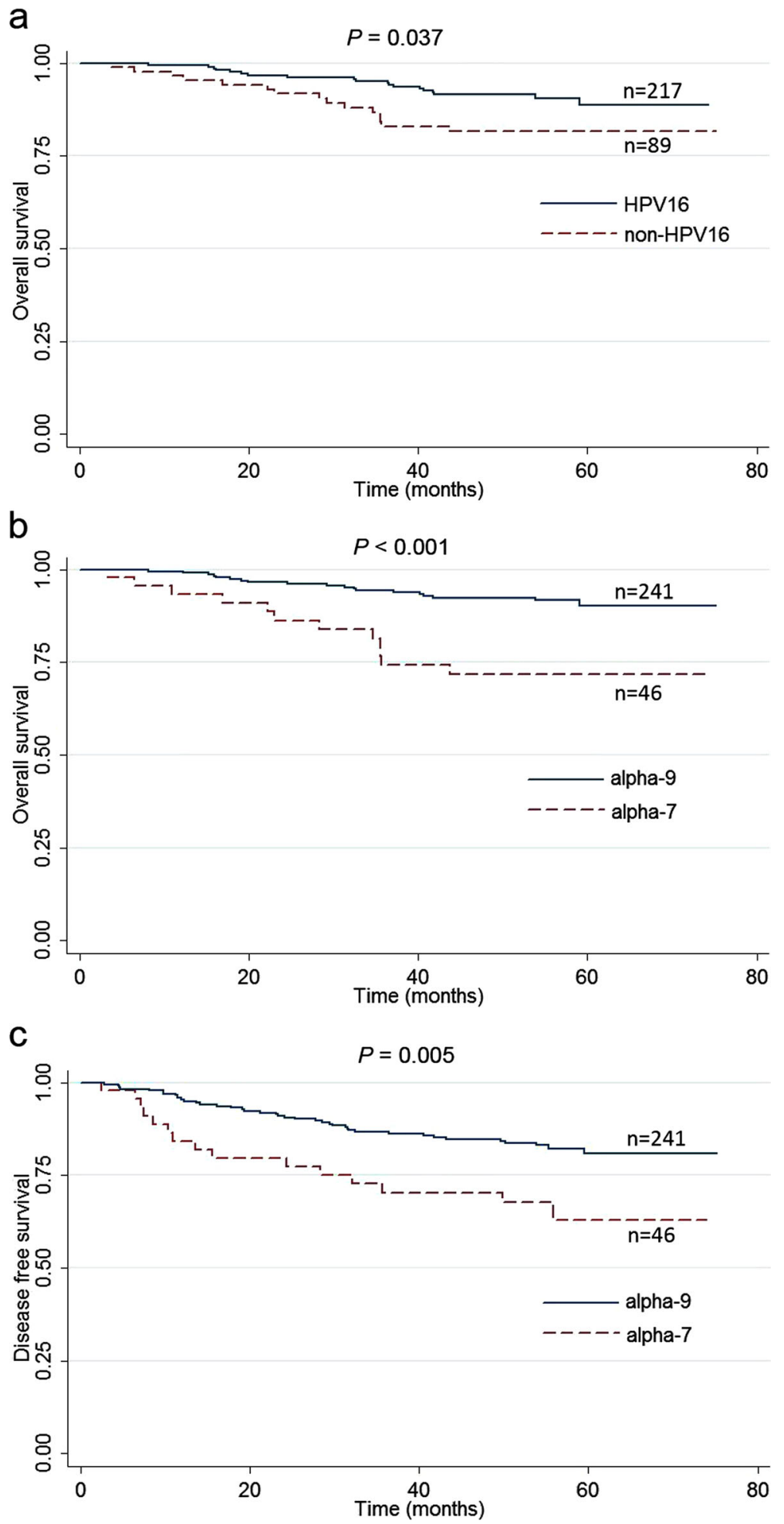

Fig. 1 The role of HPV genotype in cervical cancer prognosis. a Kaplan-Meier overall survival (OS) curves for HPV16 and non-HPV16 types; (b) Kaplan-Meier OS curves for the alpha-7 and alpha-9 species; (c) Kaplan-Meier disease free survival (DFS) curves for the alpha-7 and alpha-9 species 


\section{Conclusions}

Our results demonstrated the independent prognostic value of HPV genotype in cervical cancer. HPV genotyping could potentially help to stratify cervical cancer patients for more effective therapeutic regimens. Patients with alpha-9-caused cervical cancer may receive less aggressive therapy to reduce side effects, while those with alpha-7 positivity may require more aggressive treatment and closer monitoring. Identifying the mechanisms by which the alpha-7 species leads to a poorer prognosis could help to improve the outcome of cervical cancer patients.

\section{Additional file}

Additional file 1: Table S1. Multiple HPV infections in cervical cancer patients. This table is the supporting information for line 169-171. Table S2. Survival analysis of the alpha-9 types in cervical cancer patients. This table is the supporting information for line 193-196. Table S3. Stratified analysis of HPV genotype and cervical cancer survival. This table is the supporting information for line 198-203. (DOCX $40 \mathrm{~kb}$ )

\section{Abbreviations}

AC: Adenocarcinoma; ASC: Adeno-squamous carcinoma; CCRT: Concurrent chemoradiotherapy; CRT: Chemoradiotherapy; CT: Chemotherapy; DFS: Disease free survival; EMT: Epithelial-to-mesenchymal transition; HPV: Human papillomavirus; HR: Hazard ratio; OPC: Oropharyngeal cancer; OS: Overall survival; RT: Radiotherapy

\section{Acknowledgements}

Not applicable.

\section{Funding}

This study was supported by the National Natural Science Fund from the National Natural Science Foundation of China $(81,502,873,81,373,079$ and $81,673,265)$, Research Special Fund for Public Welfare Industry of Health (201402003), Natural Science Foundation of Jiangsu Province for Youth (BK20150997), PUMC Youth Fund (3,332,016,131 and 33,320,140,168), Natural Science Foundation of the Higher Education Institutions of Jiangsu Province (15KJB330001), Priority Academic Program for the Development of Jiangsu Higher Education Institutions (Public Health and Preventive Medicine), and Beijing Nova Program (xx2012067)

\section{Availability of data and materials}

The datasets used and analysed during the current study are available from the corresponding author on reasonable request.

\section{Authors' contributions}

$\mathrm{DH}$ performed statistical analyses and drafted the initial manuscript. MJ, JZ, and $X F$ did the experiments. HM checked statistical results. $Z L, J Y, H C$, and $Y Y$ collected the clinical data and cervical exfoliated cell samples. GJ, ZH, and HS supervised the follow-up and revised the manuscript critically. $\mathrm{KZ}, \mathrm{NL}$, and MD obtained financial support and were responsible for study design and interpretation of results. All authors read and approved the final manuscript.

\section{Competing interests}

The authors declare that they have no competing interests.

\section{Consent for publication}

Not applicable.

\section{Ethics approval and consent to participate}

This study was approved by the ethics committees of National Cancer Centre / Cancer Hospital, Chinese Academy of Medical Sciences, and written informed consent was obtained from each participant.

\section{Publisher's Note}

Springer Nature remains neutral with regard to jurisdictional claims in published maps and institutional affiliations.

\section{Author details}

${ }^{1}$ Department of Epidemiology and Biostatistics, School of Public Health, Nanjing Medical University, Nanjing 211166, China. ${ }^{2}$ Program Office for Cancer Screening in Urban China, National Cancer Centre/Cancer Hospital, Chinese Academy of Medical Sciences and Peking Union Medical College, Beijing 100021, China. ${ }^{3}$ State Key Laboratory of Reproductive Medicine, Nanjing Medical University, Nanjing 211166, China. ${ }^{4}$ Department of Cancer Prevention, National Cancer Centre/Cancer Hospital, Chinese Academy of Medical Sciences and Peking Union Medical College, Beijing 100021, China.

Received: 19 February 2017 Accepted: 15 May 2017

Published online: 05 June 2017

References

1. Torre LA, Bray F, Siegel RL, Ferlay J, Lortet-Tieulent J, Jemal A. Global cancer statistics, 2012. CA Cancer J Clin. 2015;65(2):87-108.

2. de Villiers EM. Cross-roads in the classification of papillomaviruses. Virology. 2013:445(1-2):2-10.

3. de Sanjose S, Quint WG, Alemany L, Geraets DT, Klaustermeier JE, Lloveras B, et al. Human papillomavirus genotype attribution in invasive cervical cancer: a retrospective cross-sectional worldwide study. Lancet Oncol. 2010:11(11):1048-56.

4. Pilch H, Gunzel S, Schaffer U, Tanner B, Brockerhoff $P$, Maeurer M, et al. The presence of HPV DNA in cervical cancer: correlation with clinico-pathologic parameters and prognostic significance: 10 years experience at the Department of Obstetrics and Gynecology of the Mainz University. International journal of gynecological cancer : official journal of the International Gynecological Cancer Society. 2001;11(1):39-48.

5. Lai CH, Chang CJ, Huang HJ, Hsueh S, Chao A, Yang JE, et al. Role of human papillomavirus genotype in prognosis of early-stage cervical cancer undergoing primary surgery. J Clin Oncol. 2007;25(24):3628-34.

6. Im SS, Wilczynski SP, Burger RA, Monk BJ. Early stage cervical cancers containing human papillomavirus type 18 DNA have more nodal metastasis and deeper stromal invasion. Clinical cancer research : an official journal of the American Association for Cancer Research. 2003;9(11):4145-50.

7. Huang LW, Chao SL, Hwang JL. Human papillomavirus-31-related types predict better survival in cervical carcinoma. Cancer. 2004;100(2):327-34

8. Lai HC, Sun CA, Yu MH, Chen HJ, Liu HS, Chu TY. Favorable clinical outcome of cervical cancers infected with human papilloma virus type 58 and related types. Int J Cancer. 1999:84(6):553-7.

9. Fule T, Csapo Z, Mathe M, Tatrai P, Laszlo V, Papp Z, et al. Prognostic significance of high-risk HPV status in advanced cervical cancers and pelvic lymph nodes. Gynecol Oncol. 2006;100(3):570-8.

10. Zampronha Rde A, Freitas-Junior R, Murta EF, Michelin MA, Barbaresco AA Adad SJ, et al. Human papillomavirus types 16 and 18 and the prognosis of patients with stage I cervical cancer. Clinics (Sao Paulo). 2013;68(6):809-14.

11. Song X, Sturgis EM, Huang Z, Li X, Li C, Wei Q, et al. Potentially functional variants of p14ARF are associated with HPV-positive oropharyngeal cancer patients and survival after definitive chemoradiotherapy. Carcinogenesis. 2014;35(1):62-8

12. Schuler S, Ponnath M, Engel J, Ortmann O. Ovarian epithelial tumors and reproductive factors: a systematic review. Arch Gynecol Obstet. 2013;287(6):1187-204.

13. Lai CH, Chou HH, Chang CJ, Wang CC, Hsueh S, Huang YT, et al. Clinical implications of human papillomavirus genotype in cervical adenoadenosquamous carcinoma. Eur J Cancer. 2013;49(3):633-41.

14. Hang DR, Islam SE, Sharma KH, Kuo SW, Zhang CZ, Wang JJ. Annealing effects on the optical and morphological properties of ZnO nanorods on AZO substrate by using aqueous solution method at low temperature. Nanoscale Res Lett. 2014:9(1):632.

15. Yang SH, Kong SK, Lee SH, Lim SY, Park CY. Human papillomavirus 18 as a poor prognostic factor in stage I-IIA cervical cancer following primary surgical treatment. Obstet Gynecol Sci. 2014;57(6):492-500.

16. Kang WD, Kim CH, Cho MK, Kim JW, Cho HY, Kim YH, et al. HPV-18 is a poor prognostic factor, unlike the HPV viral load, in patients with stage IB-IIA cervical cancer undergoing radical hysterectomy. Gynecol Oncol. 2011;121(3):546-50. 
17. Tong SY, Lee YS, Park JS, Namkoong SE. Human papillomavirus genotype as a prognostic factor in carcinoma of the uterine cervix. Int J Gynecol Cancer. 2007;17(6):1307-13.

18. Cuschieri K, Brewster DH, Graham C, Nicoll S, Williams AR, Murray Gl, et al. Influence of HPV type on prognosis in patients diagnosed with invasive cervical cancer. Int J Cancer. 2014;135(11):2721-6.

19. Gillison ML, Alemany L, Snijders PJ, Chaturvedi A, Steinberg BM, Schwartz S, et al. Human papillomavirus and diseases of the upper airway: head and neck cancer and respiratory papillomatosis. Vaccine. 2012;30(Suppl 5):F34-54.

20. Ang KK, Harris J, Wheeler R, Weber R, Rosenthal DI, Nguyen-Tan PF, et al. Human papillomavirus and survival of patients with oropharyngeal cancer. N Engl J Med. 2010;363(1):24-35.

21. Kreimer AR, Clifford GM, Boyle P, Franceschi S. Human papillomavirus types in head and neck squamous cell carcinomas worldwide: a systematic review. Cancer Epidemiol Biomark Prev. 2005;14(2):467-75.

22. Fakhry C, Westra WH, Li S, Cmelak A, Ridge JA, Pinto H, et al. Improved survival of patients with human papillomavirus-positive head and neck squamous cell carcinoma in a prospective clinical trial. J Natl Cancer Inst. 2008;100(4):261-9.

23. Worden FP, Kumar B, Lee JS, Wolf GT, Cordell KG, Taylor JM, et al. Chemoselection as a strategy for organ preservation in advanced oropharynx cancer: response and survival positively associated with HPV16 copy number. J Clin Oncol. 2008;26(19):3138-46.

24. Lassen P, Eriksen JG, Hamilton-Dutoit S, Tramm T, Alsner J, Overgaard J. Effect of HPV-associated p16INK4A expression on response to radiotherapy and survival in squamous cell carcinoma of the head and neck. J Clin Oncol. 2009;27(12):1992-8.

25. Arends MJ, Wyllie AH, Bird CC. Human papillomavirus type 18 is associated with less apoptosis in fibroblast tumours than human papillomavirus type 16. Br J Cancer. 1995;72(3):646-9.

26. Spanos WC, Hoover A, Harris GF, Wu S, Strand GL, Anderson ME, et al. The PDZ binding motif of human papillomavirus type 16 E6 induces PTPN13 loss, which allows anchorage-independent growth and synergizes with ras for invasive growth. J Virol. 2008;82(5):2493-500.

27. Watson RA, Thomas M, Banks L, Roberts S. Activity of the human papillomavirus E6 PDZ-binding motif correlates with an enhanced morphological transformation of immortalized human keratinocytes. J Cell Sci. 2003;116(Pt 24):4925-34.

28. Thomas M, Massimi P, Navarro C, Borg JP, Banks L. The hScrib/Dlg apicobasal control complex is differentially targeted by HPV-16 and HPV-18 E6 proteins. Oncogene. 2005;24(41):6222-30.

\section{Submit your next manuscript to BioMed Central and we will help you at every step:}

- We accept pre-submission inquiries

- Our selector tool helps you to find the most relevant journal

- We provide round the clock customer support

- Convenient online submission

- Thorough peer review

- Inclusion in PubMed and all major indexing services

- Maximum visibility for your research

Submit your manuscript at www.biomedcentral.com/submit 